\title{
PROSPECTS
}

\section{Debating the literature}

A new approach can revitalize literature updates, building skills and teamwork at the same time. B. Harihara Venkatraman, Dipankar Basak and Dhandapani Venkataraman report.

Research-group meetings, presentations, journal clubs, online blogs and literature seminars - these are the usual ways for labs to keep abreast of the scientific literature. But they often lack in-depth contributions from many participants. Postdocs and students may view literature updates as a chore rather than as a stimulating intellectual exercise.

To ensure incisive and deliberative discussions among all participants, we have pursued a new approach - adopting debates as the format for literature updates during our weekly group meetings. These debates provide not only an opportunity to learn about the topical literature, but also a way for group members to develop vital soft skills, such as working as a team and presenting data.

Our research group, undergraduates included, is divided into three sub-groups: 'pro', 'con' and 'jury'. The members switch sub-groups each week. The jury chooses a research paper published as a communication in the most recent edition of Journal of the American Chemical Society (JACS), a week before the group meeting. The pro group argues that the paper meets the publication criteria in terms of novelty, significance, broad interest and scholarly presentation. The con group argues otherwise. Both teams base their arguments on a critical analysis of the data provided in the paper and prior literature.

Each team has three minutes per speaker and three minutes for closing arguments. After closing arguments, the jury can ask questions related either to the arguments or to the paper. Each group has two minutes to answer the jury's question and to defend against the arguments made by the other group. Then jury members vote for a winner in a secret ballot. The principal investigator does not participate but offers post-debate comments on the validity of arguments made, essential points that should have been discussed and areas that need improvement.

Although each paper has already been published in JACS, the jury has historically shown no bias for the pro group. The debates have actually led us to dispute the conclusions of a few papers, including the accuracy of cartoons or figures in the papers. In a couple of cases, we contacted the principal authors to provide our post-debate viewpoints.

Through such debates, our group members have developed the ability to work as a team, dissect scientific arguments, listen to opposing viewpoints without prejudgements, overcome personal bias on a topic and provide a professional response based on the data. They have learned to engage in constructive criticism and adopt a healthy scepticism. These skills are valuable not only for their own research projects, but also in the development of students for their chosen careers.

B. Harihara Venkatraman and Dipankar Basak are graduate students in the department of chemistry at the University of Massachusetts Amherst. Dhandapani Venkataraman is their adviser.

\section{POSTDOC JOURNAL}

\section{Expect the unexpected}

Labs are often serene, orderly places. But disaster may lurk. Recently, I was at my desk, happily reading an article about how transcription remodelling factors regulate nucleosome dynamics, when I heard a commotion next door. I ran over to find that a pipe had burst. Water was spewing everywhere, flooding the lab within minutes.

Researchers abandoned their experiments as everyone worked together to staunch the inundation. Someone built a makeshift dam while others mopped up gallons and gallons of water, pouring it all down the sink. Finally, after one of us found and shut off the emergency valve, the deluge slowed and stopped. We all sighed with relief when we found that no equipment had been damaged.

That wasn't our only neardisaster. A few months ago, the $-80^{\circ} \mathrm{C}$ freezer in our lab broke, triggering a mad scramble to transfer precious antibodies, strains and reagents to a backup freezer. A lab's deep freezer often contains years of research. And then there's the terror of losing years of stored digital data to a hard-drive crash.
Last year a friend of mine had a hard-drive malfunction, lost part of his thesis and had to start again from scratch. What have I learned from these unfortunate situations? Be vigilant about protecting laboratory reagents and research data. And don't be lulled into a false sense of security by a quiet, tranquil lab bathed in the soft hum of a working freezer. Expect the unexpected.

Bryan Venters is a postdoctoral fellow at the Center for Eukaryotic Gene Regulation at Pennsylvania State University, University Park.

\section{Boon for biotech}

There's life yet for biotechnology firms, and 15 of them have special promise, according to FierceBiotech, an industry bulletin that covers the biotech sector. FierceBiotech named six firms from Greater Boston, Massachusetts; one from the United Kingdom; five from California and another three from elsewhere in the United States in its list of the year's top 15 biotechs (see www.fiercebiotech. com). The financial downturn made the cream of the crop easier to identify, FierceBiotech said, because only a select group of biotechs have thrived in the current climate. FierceBiotech based its selections on such criteria as the company's drug development experience, the novelty or efficacy of its product and the transparency of its management.

\section{Student enrolment up}

Graduate-level enrolment in science and engineering in the United States reached 516,199 in 2007 , up $3.3 \%$ on 2006 , according to the most recent survey from the National Science Foundation. The year-on-year increase was the highest since 2002 and nearly double that of the year before. Of these students, nearly threequarters $(72 \%)$ were enrolled full time and almost three quarters (71\%) were US citizens or permanent residents. More than half (56\%) were men. Among US citizens and permanent residents, men and women enrolled in almost equal numbers, but among temporary visa holders, two-thirds were men. New full-time enrolments of non-US students were up $8.3 \%$.

\section{Plain English}

Two young researchers have launched a free manuscript-editing service to help scientists from developing countries get papers published in English-language journals. Justin Chakma, a research analyst at the McLaughlin-Rotman Centre for Global Health in Toronto, Canada, and Alexander Patananan, a biochemistry doctoral student at the University of California, Los Angeles, set up SciEdit earlier this year as a spin-off of the Journal of Young Investigators, an international student-run, peer-reviewed journal. In choosing which papers to improve, it assesses the merit of the science, not just the use of English. To date, SciEdit has accepted 10 manuscripts from India, South Africa, Pakistan, China and Nepal. 